\title{
Clinical, Ultrasonographic and Histopathological Findings of Gastrointestinal Disorders in Cats
}

\author{
Christiane Aguero da Silva', Simone Carvalho dos Santos Cunha', \\ Heloisa Justen Moreira de Souza ${ }^{2}$, Amanda Chaves de Jesus ${ }^{3}$ \& Ana Maria Reis Ferreira ${ }^{1,4}$
}

\begin{abstract}
Background: Gastrointestinal disorders are common in cats, and the differentiation between inflammatory and neoplastic disease is essential to determine therapy. Therefore, ultrasonographic evaluation is an important tool for intestinal diagnosis in cats. The aim of this study is to evaluate the clinical, ultrasonographic and histopathological characteristics of cats with intestinal diseases.

Materials, Methods \& Results: Forty cats with gastrointestinal clinical signs and abdominal ultrasound findings consistent with inflammatory bowel disease or gastrointestinal neoplasia were studied. Ultrasound evaluated all abdominal organs, with emphasis on the gastrointestinal tract, and parameters included the thickness of gastric and intestinal wall, the variations of its echogenicity, reduced intestinal lumen, mesenteric lymph nodes, involvement of other abdominal organs and presence of abdominal effusion. All cats were referred to exploratory laparotomy, in order to obtain biopsy samples. Mean age was 11.6 years and there were 23 females and 17 males. Clinical signs included weight loss (87.5\%), vomiting $(82.5 \%)$, hyporexia (75\%), diarrhea (35\%), constipation (10\%), polyphagia $(5 \%)$ and intestinal gases $(5 \%)$. In abdominal ultrasound, the most common findings were increased thickness of the gastric and/or intestinal wall, decreased echogenicity of the intestinal wall, reduction of the intestinal lumen due to severe wall thickening or presence of obstructive mass, and lymphadenopathy. Histopathology revealed alimentary lymphoma (AL) in 19 cases, inflammatory bowel disease (IBD) in 10 cases, intestinal adenocarcinoma in 7 cases, mast cell tumor in 2 cases and intestinal hemangiosarcoma in 2 cases. Discussion: Weight loss and vomiting were the two main complaints of the owners. However, diarrhea, described by previous authors as one of the main clinical signs in cats with intestinal diseases, was present in only $35 \%$ of cats of this study. The intestinal segments that were presented more frequently with increased wall thickness were duodenum and jejunum, and the mean wall thickness was $0.51 \mathrm{~cm}$. Cats diagnosed with AL had mean value of duodenum wall thickness/jejunum of $0.564 \mathrm{~cm}$, while in patients with IBD the mean value was $0.462 \mathrm{~cm}$. Whereas previous authors said that ultrasonographic findings in cats with lymphocytic lymphoma are usually indistinguishable from patients with inflammatory bowel disease and that the wall thickness in both cases may be normal or increased, the analysis presented in this study was considered representative since most cases of AL corresponded to lymphocytic lymphoma. Surgery was performed on 35 cats in the study. The choice for exploratory laparotomy (instead of endoscopy for example) was to collect all layers of the intestinal segment, and it was successful, as all samples were representative and allowed the definitive diagnosis. Histopathology revealed 19 cases of alimentary lymphoma, 10 cases of inflammatory bowel disease, 7 cases of intestinal adenocarcinoma, 2 cases of intestinal mast cell tumor and 2 cases of intestinal hemangiosarcoma. The prevalence of AL over IBD has been previously discussed, but the occurrence of adenocarcinoma and mast cell tumor was higher in the present study. On feline physical examination, attention should be directed to abdominal palpation, since this study showed that $47.5 \%$ of the animals had intestinal wall thickening. The ultrasonographic evaluation and histopathological diagnosis is essential for the clinical management of cats with intestinal diseases.
\end{abstract}

Keywords: feline, intestinal, neoplasia, ultrasound, histopathology.

${ }^{1}$ Programa de Pós-Graduação em Medicina Veterinária (Clínica e Reprodução Animal). Faculdade de Veterinária. Universidade Federal Fluminense, Niterói, RJ-Brasil. ${ }^{2}$ Departamento de Medicina e Cirurgia Veterinária. Instituto de Veterinária. Universidade Federal Rural do Rio de Janeiro, Seropédica, RJ - Brasil. ${ }^{3}$ Veterinário Autônomo. Rio de Janeiro, RJ - Brasil. ${ }^{4}$ Departamento de Patologia e Clínica Veterinária. Faculdade de Veterinária. Universidade Federal Fluminense, Niterói, RJ-Brasil. CORRESPONDENCE: C.A. Silva [christianeaguero@ hotmail.com - Tel.: +55 (21) 2629-9527]. Departamento de Patologia e Clínica Veterinária, Faculdade de Veterinária, Universidade Federal Fluminense (UFF). Rua Vital Brazil Filho n. 64. Bairro Santa Rosa. CEP 24230-340 Niterói, RJ, Brazil. 
C.A. Silva, S.C.S. Cunha, H.J.M. Souza, A.C. Jesus \& A.M.R. Ferreira. 2018. Clinical, Ultrasonographic and Histopathological Findings of Gastrointestinal Disorders in Cats.

Acta Scientiae Veterinariae. 46: 1591.

\section{INTRODUCTION}

Alimentary lymphoma, adenocarcinoma, and mast cell tumors are the most common intestinal tumors in cats. AL is characterized by lymphoid infiltration of the stomach and/or intestines wall, with or without lymph node involvement. Diffuse small cell lymphoma, considered as a low grade lymphoma, is the most common histological type, whereas large cell lymphoma, a high grade lymphoma, is considered more aggressive and usually involves the mesenteric lymph nodes. Inflammatory bowel disease is another common intestinal disorder in cats and can be confused with small cell intestinal lymphoma, as both cause similar clinical signs and histological findings [2,3,5].

The differentiation between inflammatory and neoplastic disease is crucial to the proper choice of therapy $[5,7]$. Therefore, the ultrasonographic evaluation is an important tool for intestinal chronic diseases diagnosis in cats. It is a noninvasive method for evaluation of parameters, such as intestinal wall thickening, wall layers and motility. Changes in echogenicity of intestinal wall layers and luminal contents can also be evaluated $[1,8,10]$.

The aim of this study is to evaluate the clinical, ultrasonographic and histopathological characteristics of cats with intestinal diseases.

\section{MATERIALS AND METHODS}

\section{Animals}

Forty cats with intestinal disease were studied. The cats treated were included in the study, regardless of gender, sexual status and breed. Inclusion criteria were cats with intestinal diseases, including animals with feline leukemia virus (FeLV), which had persistent clinical symptoms for more than three weeks. Animals previously submitted to chemotherapy were excluded from the study.

History information, including appetite, weight maintenance, vomiting, diarrhea, constipation, deworming, food diet and previous treatments were collected. Physical examination evaluated hydration, mucous membranes, hair coat, nutritional status, rectal temperature and body weight. Abdominal palpation was performed to evaluate the presence of intestinal thickening, abdominal masses, intestinal gas, dry stools, lymphadenopathy and hepato-splenomegaly. Thoracic radiographs were performed in all cats for metastasis evaluation, as well as echocardiography.
Haematological and ultrasound evaluation

Haematological and biochemical evaluation included blood count, BUN, creatinine, alanine aminotransferase, potassium, albumin, FIV/FeLV serology, blood glucose and serum total $\mathrm{T}_{4}$. Fecal examination was also performed in all cats.

Ultrasound evaluated all abdominal organs, with emphasis on the gastrointestinal tract. The parameters included the thickness of gastric and intestinal wall, and variations of its echogenicity, reduced intestinal lumen (due to severe wall thickening or presence of masses causing partial and/or total intestinal obstruction), mesenteric lymph nodes, presence of intestinal gas, dry stool in the colon, involvement of other abdominal organs, presence of abdominal effusion, peritonitis and adhesions. Wall thickness was considered normal if $\leq 0.22 \mathrm{~cm}$ in stomach [10], $\leq$ $0.28 \mathrm{~cm}$ in intestinal wall of the duodenum and jejunum, $\leq 0.32 \mathrm{~cm}$ in ileum and $\leq 0.17 \mathrm{~cm}$ in colon [16]. Mesenteric lymph nodes were considered normal if $\leq$ $0.5 \mathrm{~cm}$. The spleen was evaluated for its echogenicity, echotexture and presence of nodules, and splenomegaly was considered if spleen passed the caudal edge of the left kidney, the midline toward the left epigastric region and umbilicus. The liver was evaluated for its echogenicity, presence of masses, vessels and edges, and hepatomegaly was considered when its cranial limit exceeded sixth intercostal space and caudal limit exceeded 11th intercostal space. The equipment used was Logiq P5 GE and images were formed in a frequency range from 7.5 to $11 \mathrm{MHz}$ using a linear transducer.

Surgery

All cats were referred to exploratory laparotomy, in order to obtain biopsy samples for histopathology. Acepromazine $\left(\text { Acepram }^{\circledR}\right)^{1}$ at a dose of 0.03 $\mathrm{mg} \mathrm{kg}^{-1}$ intramuscularly, and meperidine $\left(\text { Dolosal }^{\circledR}\right)^{2}$ 2-4 $\mathrm{mg} \mathrm{kg}^{-1}$ intramuscularly were administered to patients with no evidence of systemic diseases. Propofol $\left(\text { Propofol }^{\circledR}\right)^{3} 4 \mathrm{mg} \mathrm{kg}^{-1}$ intravenously was administered for anesthetic induction, and maintenance was done with isoflurane $\left(\text { Forane }^{\circledR}\right)^{4}$ and $100 \%$ oxygen. In cats with heart disease, premedication was done with meperidine ${ }^{2}$ (2-4 $\mathrm{mg} \mathrm{kg}^{-1}$ intramuscularly and anesthetic induction with etomidate (Etomidato $\left.{ }^{\circledR}\right)^{2} 2 \mathrm{mg} \mathrm{kg}^{-1}$ intravenously. An incision in the pre-retroumbilical middle line was performed. An incision of one centimeter in the thickened intestinal segment was performed, and biopsy samples were collected. The intestine was su- 
tured with 4-0 polypropylene, with simple interrupted suture pattern. In cases of diffuse intestinal disease, two fragments were collected in different segments of the intestine. If lymph nodes, pancreas or liver were increased or had nodules, biopsy samples were also taken from these organs. In cases of intestinal masses causing obstruction, enteroanastomosis with $3 \mathrm{~cm}$ margin was performed.

After surgery, the cats remained hospitalized for $72 \mathrm{~h}$, and food was not offered in the first $24 \mathrm{~h}$. Pain management included meloxicam $\left(\text { Maxicam }^{\circledR}\right)^{5}$ $0.1 \mathrm{mg} \mathrm{kg}^{-1}$ subcutaneously SID for 4 days, tramadol $\left(\text { Cronidor }^{\circledR}\right)^{6} 2 \mathrm{mg} \mathrm{kg}^{-1}$ subcutaneously BID for 5 days and dipyrone (Novalgina $\left.{ }^{\circledR}\right)^{7} 25 \mathrm{mg} \mathrm{kg}^{-1}$ subcutaneously BID for 5 days. Antibiotics included ampicillin $\left(\text { Ampicilina }{ }^{\circledR}\right)^{8} 10 \mathrm{mg} \mathrm{kg}^{-1}$ intravenously every $8 \mathrm{~h}$ and metronidazole $\left(\text { Flagyl }^{\circledR}\right)^{7} 10 \mathrm{mg} \mathrm{kg}^{-1}$ intravenously BID, which were continued orally by owners at home. Skin suture removal and antibiotics interruption were performed after 10 days.

\section{Histopathological examination}

All biopsy samples were fixed in buffered formalin $10 \%$, processed and included in paraffin. Histological sections were $5 \mu \mathrm{m}$ thick and were subsequently stained with hematoxylin and eosin. The evaluated parameters were type of cell infiltrate, cell predominance, cell infiltration distribution, degree of invasion of infiltrates (submucosa/transmural), presence of mucosal edema, mucosal architecture (fibrosis and morphological changes of intestinal villi), degree of cell atypias and degree of cell proliferation. Mesenteric lymph nodes cytoarchitecture and presence of inflammatory and neoplastic infiltrates were also evaluated.

\section{Statistical analysis}

Statistical analysis was performed, with categorical variables described by proportions and numerical variables described by parameters (mean, standard deviation, minimum and maximum values, median and interquartile ranges). The normality of the numerical data was assessed using the ShapiroWilk test. Comparison of two numerical data sets was performed by Student's $t$ test. The comparison of equal variances between two groups of data was performed by the Levene test. When the two groups did not meet the normal criteria, comparison between them was carried out by the nonparametric MannWhitney. Simultaneous comparison of more than two numeric data groups that did not satisfy the criteria of normality was performed using the Kruskal-Wallis test. Evaluation of association between categorical variables was preceded by the chi- square test or, in the case of cross-tables with two categories each, by Fisher 's exact test. Comparisons between proportions of categories were performed using chi-square test or, in the case of dichotomous variables, with Fisher's exact test. Significance level was $\alpha=0.05$, therefore differences were considered statistically significant when $p$-values were less than 0.05 .

\section{RESULTS}

Mean age was $11.6 \pm 3.29$ years. Weight ranged from 1.77 to $6.65 \mathrm{~kg}$, with a mean of $4.158 \pm 1.2756$. Mixed breed represented $87.5 \%$ of the study population, while $7.5 \%$ were siamese and $5 \%$ were persian. There were $23(57.5 \%)$ females (all spayed) and 17 (42.5\%) males, only 2 of them were intact.

Clinical signs included weight loss in 35/40 cats $(87.5 \%)$, vomiting in $33 / 40$ cats $(82.5 \%)$ and hyporexia in $30 / 40$ cats $(75 \%)$. Only $35 \%$ of animals had diarrhea, $10 \%$ constipation, $5 \%$ polyphagia and $5 \%$ intestinal gases according to their owners.

On physical examination, 35/40 animals $(87.5 \%)$ had normal mucous membranes, 33/40 animals $(82.5 \%)$ had no coat abnormalities (such as alopecia or hypotrichosis), 29/40 animals (72.5\%) had some degree of dehydration, 19/40 animals (47.5\%) were very thin or cachectic and 4/40 animals (10\%) had elevated rectal temperature. In abdominal palpation, $47.5 \%$ of the cats had thickening of the bowel, $22.5 \%$ had intestinal crepitus, $17.5 \%$ had lymphadenopathy, $15 \%$ had hard stools, $7.5 \%$ abdominal mass, $5 \%$ had hepatomegaly and $2.5 \%$ had splenomegaly.

In abdominal ultrasonographic evaluation, 6 animals had increased thickness of the gastric wall, with a mean value of $0.55 \pm 0.24 \mathrm{~cm}$ (normal $\leq 0.22 \mathrm{~cm}$ ); 15 animals had decreased echogenicity of the intestinal wall; 37 animals had increased thickness of the intestinal wall (Figure 1), while 34 animals had increased thickness in duodenum and jejunum, with a mean value of $0.51 \pm 0.24 \mathrm{~cm}$ (normal $\leq 0.28 \mathrm{~cm}$ ), 3 animals had increased wall thickness in ileum, with an average of $0.8 \pm 0.18 \mathrm{~cm}$ (normal $\leq 0.32 \mathrm{~cm}$ ) and 4 animals had an increase in thickness of the colon wall, with an average of $0.8 \pm 0.16 \mathrm{~cm}$ (normal $\leq 0.17 \mathrm{~cm}$ ). Only 1 cat had duodenum, jejunum and ileum wall thickening 
simultaneously and 4 cats had simultaneous thickening of the duodenum, jejunum and colon. Three animals had no intestinal wall thickening in any segment. Ten cats had reduction of the intestinal lumen due to severe wall thickening or presence of obstructive mass.

Regarding the increase in mesenteric lymph nodes (normal $\leq 0.5 \mathrm{~cm}$ ), 20 cats had lymphadenopathy, which represented $50 \%$ of the studied cats (Figure 1). Intestinal gas was present in 10 cats, and hard stools in the colon were found in 4 animals. Three animals had a mass in stomach wall, measuring from $2.6 \times 1.8$ $\mathrm{cm}$ to $5.25 \times 3.25 \mathrm{~cm}$. Seven animals had intestinal masses, 4 in duodenum segments and jejunum, with the largest measuring $5.0 \times 3.5 \mathrm{~cm}, 1$ cat had two masses in ileal segments (measuring 5.0 x $2.2 \mathrm{~cm}$ and $3.7 \times 1.2$ $\mathrm{cm})$, while 2 cats presented masses in ileum-cecumcolic valve that measured $4.0 \times 2.7 \mathrm{~cm}$ and $5.0 \times 2.0$ $\mathrm{cm}$ respectively. Ten cats had liver involvement, 1 cat had simultaneous involvement of the liver and spleen, 1 cat presented splenic abnormalities and 1 cat had pancreatic abnormalities.

Five animals of the study have not undergone the surgical procedure and biopsy samples were collected by necropsy. One of these animals had an intestinal tumor with metastasis to pancreas, liver, lymph nodes and lungs. In the other 35 cats submitted to surgery (Figure 2), 2 animals died in the immediate postoperative period, 1 had septic peritonitis and the other had pneumoperitoneum caused by disruption of the gastric wall. Enteroanastomosis technique was performed on 10 animals.

Histopathology revealed AL in 19 cases, IBD in 10 cases (Figure 3), adenocarcinoma in 7 cases, mast cell tumor in 2 cases and hemangiosarcoma in two cats.

Regarding ultrasonographic evaluation, there was no statistical significance between AL and IBD $(P>0.05)$. The Mann-Whitney test indicated evidence of a statistically significant difference $(P<0.05)$ between the increased wall thickness of the duodenum of cats with AL and cats with IBD, with higher values for cases of lymphoma $(\mathrm{U}=39.5 ; P$-value $=0.031)$. There was no statistically significant difference $(P$ $>0.05$ ) in abdominal ultrasound between AL, IBD, adenocarcinoma, hemangiosarcoma and mast cell tumor. Kruskal-Wallis test indicates lack of statistically significant evidence $(P>0.05)$ between the increased thickness of the wall of the duodenum $(\mathrm{M}=7,311$; g.l. $=4 ; P$-value $=0.120)$ and ileum $(\mathrm{H}=2,000 ;$ g.l. $=2$; $P$-value $=0.368)$ classified by diagnosis.

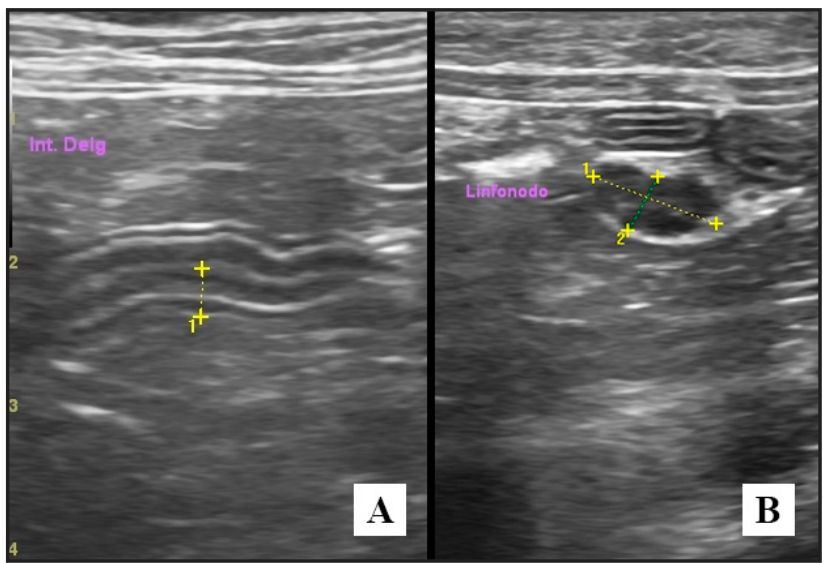

Figure 1. Ultrasonographic image of intestinal segment in a cat. A- Longitudinal image of the duodenum with increased wall thickness $(0.37 \mathrm{~cm})$. B- Enlarged mesenteric lymph node $(1.4 \times 0.6 \mathrm{~cm})$.

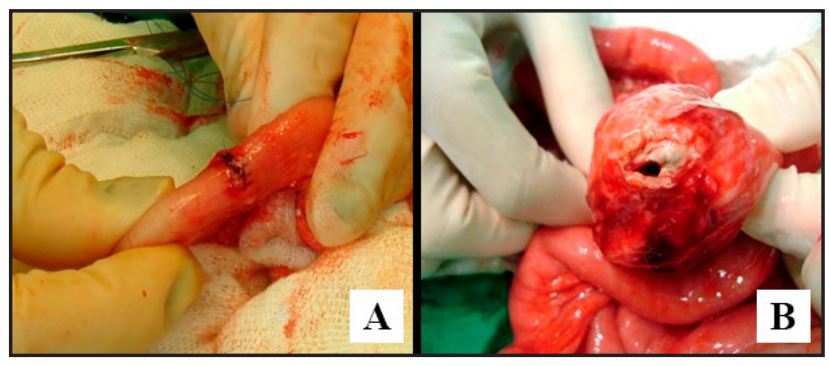

Figure 2. A- Biopsy sample collected by enterotomy. B- Perforated intestinal mass of a cat with intestinal lymphoma.

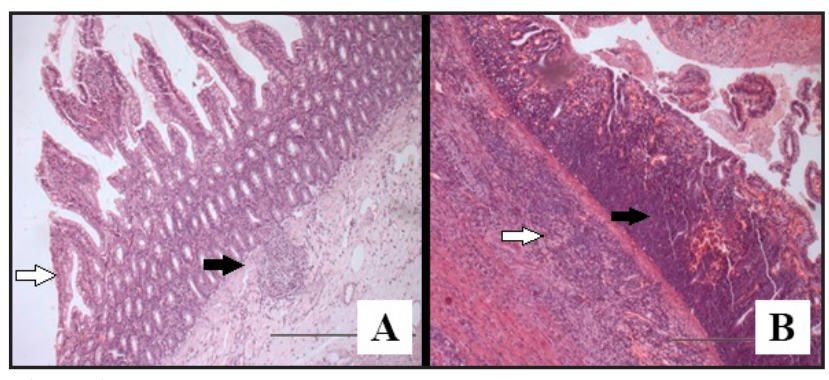

Figure 3. Histopathology of intestinal disorders in cats. A- Inflammatory Bowel Disease (IBD). Mucosal lymphocytic infiltration, with flattening of the intestinal villi (white arrow) and lymphoid follicle in submucosa (black arrow). H.E. [Bar $=50 \mu \mathrm{m}]$. B- Intestinal lymphoma. Diffuse neoplastic lymphocytic infiltrate in submucosa (white arrow) and mucosa (black arrow), with loss of architecture. H.E. [Bar $=50 \mu \mathrm{m}]$.

\section{DISCUSSION}

Cats with intestinal diseases in this study had ages ranging from 3 to 19 years old and $52.5 \%$ of cats had 12-15 years. The mean age of cats with AL was 14.5 year, more than 13 years previously described [9]. Most cats were mixed breed (35), 3 animals were siamese and 2 Persian, and there were 23 females and 17 males. Most authors believe that there is no predilection for breed or gender.

Sporadic vomiting was often considered normal by the owners, as an attempt to eliminate hairballs, 
the same reported previously [17]. The weight loss, vomiting and loss of appetite were present in $87.5 \%$, $75 \%$ and $75 \%$ cats, similarly to previous authors $[7,12,14,15,17]$. However, diarrhea, described as one of the main clinical signs in cats with intestinal diseases, was present in only $35 \%$ of cats of this study.

In one study, $20-30 \%$ of cats diagnosed with lymphoma had abdominal masses, attributed to mesenteric lymph nodes enlargement or focal intestinal masses. This author also considered that animals with high-grade lymphoma or intermediate grade lymphoma may have focal masses involving intestinal thickening and/or extra-intestinal lesions (such as mesenteric lymphadenopathy, hepatomegaly and renomegaly) [2]. In the present study, $47.5 \%$ of the cats had intestinal wall thickening on abdominal palpation, $17.5 \%$ lymphadenopathy, $7.5 \%$ abdominal masses, $7.5 \%$ hepatomegaly, similarly to previous literature. No animal presented with renomegaly but $2.5 \%$ had splenomegaly, and a hypothesis for this event would be the animals diagnosed with mast cell tumor and intestinal hemangiosarcoma occasionally seep into the spleen [13].

Ultrasonographic evaluation is the most important diagnostic exam for animals with intestinal diseases. A previous study reported that although the ultrasonographic appearance of inflammatory lesions and neoplastic infiltration are similar, the knowledge of imaging features help to recognize the characteristics of each disease [8]. All cats of this study underwent the ultrasonographic evaluation of the abdominal cavity. Cats diagnosed with alimentary lymphoma had mean value of duodenum wall thickness/jejunum of 0.564 $\mathrm{cm}$, while in patients with IBD the mean value was $0.462 \mathrm{~cm}$. The statistical analysis considered the presence of significance for the comparison of duodenal wall thickening/jejunum between animals with AL and IBD. Whereas previous studies found that ultrasono- graphic findings in cats with lymphocytic lymphoma are usually indistinguishable from patients with IBD and that the wall thickness in both cases may be normal or increased $[4,15]$, the analysis presented in this study was considered representative since most cases of AL corresponded to lymphocytic lymphoma.

\section{CONCLUSION}

The main complaints of owners of cats with gastrointestinal diseases were vomiting and weight loss. Dehydration, low weight and intestinal wall thickening in the abdominal palpation were the most frequent findings during the physical examination of these cats. Gastrointestinal lymphoma and inflammatory bowel disease were the most frequent histopathological diagnosis. The ultrasonographic evaluation and histopathological diagnosis is essential for the clinical management of cats with intestinal diseases.

\section{MANUFACTURERS}

1Vetnil Indústria e Comércio de Produtos Veterinários Ltda. Louveira, SP, Brazil.

${ }^{2}$ Cristália Produtos Químicos e Farmacêuticos. Rio de Janeiro,

RJ, Brazil.

${ }^{3}$ Biosintética Laboratório. São Paulo, SP, Brazil.

${ }^{4}$ Abbott Laboratórios Brasil Ltda. São Paulo, SP, Brazil.

${ }^{5}$ Ourofino Saúde Animal Participações. Cravinhos, São Paulo, SP, Brazil.

${ }^{6}$ Agener União Saúde Animal. São Paulo, SP, Brazil.

${ }^{7}$ Sanofi-Aventis Farmacêutica. Suzano, SP, Brazil.

${ }^{9}$ Medley Indústria Farmacêutica Ltda. Campinas, SP, Brazil.

Funding. The funding for this research was provided by CNPq (Conselho Nacional de Desenvolvimento Científico e Tecnológico).

Ethical approval. This study was submitted and approved by the Ethics Committee on Animal Use (CEUA) of the Fluminense Federal University (n. 683).

Declaration of interest. The authors declared no potential conflicts of interest with respect to the research, authorship, and/or publication of this article.

\section{REFERENCES}

1 Baez J.L., Hendrick M.J. \& Walker L.M. 1999. Radiographic, ultrassonographic, and endoscopic findings in cats with inflammatory bowel disease of the stomach and small intestine: 33 cases (1990-1997). Journal of American Veterinary Medical Association. 215(3): 349-354.

2 Barrs V.R. \& Beatty J.A. 2012. Feline Alimentary Lymphoma: 1. Classification, risk factors, clinical signs and noninvasive diagnostic. Journal of Feline Medicine and Surgery. 14(3): 182-190.

3 Briscoe K.A., Krockeenberger M. \& Beatty J.A. 2011. Histopathologycal and Immunohistochemical Evaluation of 53 Cases of Feline Lymphoplasmacytic Enteritis and Low-Grade Alimentary Lymphoma. Journal of Comparative Pathology. 145(2): 187-198.

4 Carreras J.K., Goldschmidt M., Lamb M., Mclear R.C., Drobatz K.J. \& Sorenmo K.U. 2003. Feline ephiteliotro- 
C.A. Silva, S.C.S. Cunha, H.J.M. Souza, A.C. Jesus \& A.M.R. Ferreira. 2018. Clinical, Ultrasonographic and Histopathological Findings of Gastrointestinal Disorders in Cats. Acta Scientiae Veterinariae. 46: 1591.

pic intestinal malignant lymphoma: 10 cases (1997-2000). Journal of Veterinary Internal Medicine. 17(3): 326-331.

5 Crystal M.A. 2004. Doença Intestinal Inflamatória In: Norsworthy G.D., Crystal M.A., Grace S.F. \& Tilley L.P. (Eds). O Paciente Felino. SãoPaulo: Manole, pp.356-362.

6 Evans S.E., Bonczynski J.J., Broussard J.D., Ham E. \& Baer K.E. 2006. Comparison of endoscopic and full-thickness biopsy specimens for diagnosis of inflammatory bowel disease and alimentary tract lymphoma in cats. Journal of American Veterinary Medical Association. 229(9): 1447-1450.

7 Fondacaro J.V., Richter K.P., Carpenter J.L., Hart J.R., Hill S.L. \& Fettman M.J. 1999. Feline gastrointestinal lymphoma: 67 cases (1988-1996). European Journal of Comparative Gastroenterology. 4(2): 5-11.

8 Gaschen L. 2011. Ultrasonography of Small Intestinal Inflamatory and Neoplastic Disease in Dogs and Cats. Veterinary Clinics of North America: Small Animal Practice. 41(2): 329-344.

9 Gieger T. 2011. Alimentary Lymphoma in Cats and Dogs. Veterinary Clinics of North America: Small Animal Practice. 41(2): 419-432.

10 Goggin J.M., Biller D.S., Debey B.M., Pickar J.G. \& Mason D. 2000. Ultrassonographic measurement of gastrointestinal wall thickness and the ultrasonographic appearance of the ileo-colic in healthy cats. Journal of American Animal Hospital Association. 36(3): 224-228.

11 Jergens A.E. \& Crandell J.N. 2006. Clinical Stating for Inflamatory Bowel Disease In: August J.R. (Ed). Consultations in Feline Internal Medicine. Philadelphia: WB Saunders Company, pp.127-132

12 Jergens A.E. 2012. Feline idiopathic inflammatory bowel disease: What we know and what remains to be unraveled. Journal of Feline Medicine and Surgery. 14(7): 445-458.

13 Laurenson M.P., Skorupski K.A., Moore P.F. \& Zwingenberger A.L. 2011. Ultrasonographic of intestinal mast cell tumors in the cat. Veterinary Radiology and Ultrasound. 52(3): 330-334.

14 Lecoindre P. \& Chevallier M. 1997. Contribuition to the study of feline inflammatory bowel disease: 51 cases (19911994). Revue de Médecine Vétérinaire. 148(11): 893-902.

15 Lingard A.E., Briscoe K. \& Beatty J.A. 2009. Low-grade alimentary lymphoma: clinicopathological findings and response to treatment in 17 cases. Journal of Feline Medicine and Surgery. 11(8): 692-700.

16 Newell S.M., Graham J.P., Roberts G.D., Ginn P.E. \& Harrison J.M. 1999. Sonography of the normal feline gastrointestinal tract. Veterinary Radiology and Ultrasound. 40(1): 40-43.

17 Norsworthy G.D., Scot J., Kiupel M., Olson J.C. \& Gassler L.N. 2013. Diagnosis of chronic small bowel disease in cats: 100 cases (2008-2012). Journal of Veterinary Medical Association. 243(10): 1455-1461.

18 Trepanier L. 2009. Idiopathic Inflammatory Bowel Disease in Cats: Rational treatment selection. Journal of Feline Medicine and Surgery. 11(1): 32-38. 